\title{
Women in Science: What Are the Barriers for Women in Senior Career Positions
}

\author{
Olfa Messaoud1, Rana Dajani2,3 \\ ${ }^{1}$ Biomedical Genomics and Oncogenetics Laboratory, Institut Pasteur de Tunis, University of Tunis El Manar, Tunis, Tunisia \\ ${ }^{2}$ Department of Biology and Biotechnology, Faculty of Science, The Hashemite University, Zarqa, Jordan \\ ${ }^{3}$ Jepson School of Leadership, University of Richmond, Richmond, USA \\ Email: olfa.messaoud@pasteur.tn
}

How to cite this paper: Messaoud, O., \& Dajani, R. (2021). Women in Science: What Are the Barriers for Women in Senior Career Positions. Advances in Applied Sociology, 11, 492-499.

https://doi.org/10.4236/aasoci.2021.1110043

Received: August 17, 2021

Accepted: October 25, 2021

Published: October 28, 2021

Copyright $\odot 2021$ by author(s) and Scientific Research Publishing Inc. This work is licensed under the Creative Commons Attribution International License (CC BY 4.0).

http://creativecommons.org/licenses/by/4.0/

\begin{abstract}
For several years, many governmental and non-governmental agencies, as well as researchers have made great efforts in addressing issues related to the factors that prevent women from pursuing STEM (Science, Technology, Engineering and Mathematics) careers. These efforts resulted in better representation of women in these fields both in the Arab countries as well as in other developed countries. However, in these countries, women remain under-represented in senior career positions, e.g., ministers, members of the parliament, heads of state institutions. Here, we try to identify the most important barriers that women are facing and suggest some recommendations to overcome them.
\end{abstract}

\section{Keywords}

Women in Science, Senior Positions, Universal/Specific Barriers, Environment and Psychological Obstacles, Recommendations

\section{Introduction}

Women have a fundamental role not only as caregivers, but also as members of society via their professional work and/or their social activities. Indeed, even if we speak about changing of women's roles in this century, it's clear that whatever the cultural context and the socio-economic level of the family, the woman is always considered as the primary caregiver for her family. Thus, she is driven by her instinct as well as social norms to dedicate more time than any other family members to responding to the daily life needs of her home and looking after her children whatever their ages. Women who have children or care for elderly family members are particularly struggling to reach not just harmony but perfection 
in their motherhood and professional roles. Although there has been a slight shift in attitudes of males in taking up caregiving roles, this is yet not the norm in many countries.

At the professional level, a large slogan of gender balance and better representation of women has been widely looked forward to by almost all communities and in different work fields. However, the actual situation is still far from it. Several researches have been conducted to find out the origins of such an imbalance at a large or focused level (Ceci et al., 2009).

In this article, we focus on barriers that prevent women from reaching senior positions. The ideas presented below summarize what has been discussed during a panel discussion organized by the Arab Science Week, August 2020. Therefore, some facts and data are taken from the bibliography while others are findings and experiences reported by the women scientists (Prof. Rana Dajani (moderator); Dr. Olfa Messaoud, Dr. Alia Abukiwan, and Dr. Rana Bilbeisi (panelists)) that participated in the panel

(https://www.youtube.com/watch?v=sRuw3Y2mMCU\&list=PLkk_rfOJg7m3aZ4 59bgQ3ArPGEiA2O-v-\&index=15).

\section{Overview of the Main Barriers}

If we look at the percentage of women graduating from scientific fields of higher education in STEM and the unemployment rate for female tertiary graduates, we can easily notice a large disproportion. For example, on one side, according to a ranking published by the World Bank in May 2019, covering 114 countries during the period from 2015 to 2017, Tunisia ranks second in the world with 58\% of women graduating in scientific fields ${ }^{1}$. This is not exceptional since several Arab countries have similar or higher proportions of females compared to males e.g., Algeria (55\%), the Sultanate of Oman (53\%), Syria (50\%), etc. Here, it is important to note that the number of men graduates from STEM fields was higher than that of women in 107 countries, according to the same classification carried out by the World Bank, referring to the latest statistics from the census institute of UNESCO. These high percentages reflect absence of any type of societal patriarchal attitudes that could refrain women from incorporating the university and having a postgraduate diploma. On the other side and for the same country, $\mathrm{Tu}$ nisia, the unemployment rate for female tertiary graduates is about double the unemployment rate for male tertiary graduates. Indeed, the national population employment survey of the $3^{\text {rd }}$ quarter of 2015 showed that the unemployment rate for women with higher education qualifications is estimated at $41.1 \%$, while for men this rate is $21.4 \%$. A big proportion of them have a post-graduate diploma (master or thesis) and nearly $70 \%$ of $\mathrm{PhD}$ holders are unemployed ${ }^{2}$.

Several studies that focused on gender balance within different fields of work have been conducted and nearly all concluded that gender equity imbalance is still a predominant feature. For example, in the field of electrocardiology where are wo-

${ }^{1}$ https://databank.worldbank.org/source/gender-statistics

${ }^{2}$ https://www.tunisienumerique.com/tunisie-pres-de-70-titulaires-dun-doctorat-chomage/ 
men largely under-represented both in academic productivity and active participation in the most prominent society; the international society of electrcardiology as a president or a board constituent. This male dominance that ranges from $75 \%$ to $100 \%$ sparked a study aimed at understanding the causes behind such inequity in order to remedy it (Clarke Whalen et al., 2017). A subsequent study focusing on sex disparities in authorship order of cardiology scientific publications showed that despite that female representation has increased over the past 4 decades, women are still under-represented in senior authorship positions (Ouyang et al., 2018). According to a recent study aiming at evaluating the improvement of gender balance in pharmacy leadership in Australia, the authors predicted that we should wait a decade to achieve an average of $50 \%$ of women representation in leadership positions (Martin et al., 2021).

One of the barriers that restrains women from senior positions is poor time management. Indeed, reaching a personal-professional balance is not an easy task, it requires good management of family responsibilities along with the enormous academic responsibilities required from a senior position. In the context of Arab countries, the family stability revolves around the woman. She holds the major responsibility in educating the children, in supporting her spouse and in taking care of her parents and her parents-in-law. So, with these persistent responsibilities, even unemployed women are never really jobless. Such expectations from society can prevent women from dreaming to climb high in their career and apply for senior positions even if they have the necessary skills. In addition, time management should be considered in the work context itself. Unfortunately, in lowand middle-income countries with limited resources dedicated to research, scientists especially females are often overwhelmed with administrative tasks. This is well illustrated by women who have moved from their home to other countries with different work conditions (having project managers, less administrative tasks, big facilities), we can easily notice that as soon as they change the workplace, their careers progress at a different speed.

In addition to work conditions, under-developed countries generally lack mentorship programs that focus on leadership skills (Dajani, 2012). Indeed, leadership skills are valuable to create impact in changing culture and any behavioral feature related to gender ranging from unfairness to sexual harassment (Córdova, 2020).

Besides time management, we also point out the importance of effort management throughout the career. Unfortunately, the global schema of academies and funding organizations are oriented towards women under 40 years. This is a male centric approach that is based on the assumption that a person should be successful before the age of 40 . This does not take into consideration that a woman may get pregnant and have children and take time off because her window of having children is under 40 . Therefore, females strive to fulfill program criteria to benefit from several opportunities as long as they are eligible such as membership in scientific academies and different consortia, as well as being actively engaged in civil society and politics to have a decision-making power. This irra- 
tional overuse of her efforts in a short time period usually leads to burnout and sometimes to the premature end of her career even before it starts shining. Women who have succumbed to these pressures of becoming super achievers realize at the age of 50 when they become ready to dive into the world of senior positions, that they have to choose between continuing in senior positions or focusing on their families if they have not already lost their partners. Here, the sense of guilt and instinct play a big role, leading women to choose family which is in a great need of her presence and support, especially her parents that become old enough to ask for her attention and care. This trend can be observed in Arab countries because they are family-oriented societies.

One universal reason for lack of women in senior positions is related to patriarchal history where women were considered by many cultures as subordinates to men, thus forcing them to believe that they hold a secondary place. This could explain why some women stop themselves from dreaming to reach senior and critical decision-making positions. This is well illustrated by job negotiations; indeed, men usually confidently ask for big salaries while women not being the main breadwinners for their families generally ask for relatively lower salaries. Definitely, we see this trend in the private sector in many countries around the world even those who are considered developed such as the US, where females and males are not equally payed and do not have the same remuneration options.

Despite what has been said above, many employers prefer to have a well-paid man under the pretext they do not ask for a maternity leave neither for frequent leaves related to pregnancy or child care. This is another universal important factor to be considered as a major barrier for women gaining senior positions.

We should not forget to mention the psychological obstacle which is a universal barrier too. In fact, senior positions require great commitment in terms of time and energy, something that women generally prefer to dedicate to her family. In addition, although success may be portrayed as an agreed upon definition by society, men and women perceive success differently. For men, success is highly related to the work however, for women having a happy family with well-educated children and a good diploma that guarantees her place in society is her version of success.

Besides universal barriers, other more specific and cultural-related ones do exist. If we have to cite one specific obstacle related to the cultural context, we would like to highlight regionalism or racism behavior towards those with different backgrounds, e.g., languages, religions, etc. To that regards, one of the authors cannot forget the offensive gaze of the customs police each time she visits a European country and she asks herself is it because I am Arab or because I am Muslim and I wear a veil. As scientists, we want to keep abreast of the latest science and technology so we attend courses or trainings abroad. These opportunities are very important in getting to know other scientists and build collaborative projects with them. In these circumstances, unfortunately, socializing with other 
colleagues or the teaching or the managerial staff is usually done during the evenings around a dinner in the bar or a dancing party. Hence, building new relations becomes more than difficult for a Muslim scientist because she/he does not go to these places on principles based on religion. We find ourselves in many occasions having to put in extra-effort to gain respect and benefit from the scientific event without giving up on our principles. These challenges facing Muslims could be perceived as obstacles to international integration by some but for others they could be an incentive to work harder not only to succeed but also to shine within the scientific community thereby breaking stereotypes presented in the media.

\section{Recommendations to Overcome Barriers}

To overcome these barriers, we believe that a multi-actor policy is needed and should take into consideration the social, psychological and administrative framework.

Women are highly encouraged to learn how to manage their time in order to guarantee an equilibrium between the mother, the spouse and the scientist. This needs, in many cases a suitable mentoring framework. Society should encourage men to take a more active role in caregiving. People are inspired by successful role models. Thus, women in senior positions are highly encouraged to document their pathway and share their stories.

In order to support and encourage institutions with high percentage of senior women, we suggest the creation of an index of excellence that documents such percentages. Such an index can be used by funders as part of their evaluation of applicants from organizations.

In the same context, funding agencies should revise their criteria and attribute favorable points to older women taking into consideration time spent during pregnancy and child care. At the same time, we stress that funding programs should always maintain a high level of quality in terms of competencies and skills. The increase of senior female scientists of high caliber is exemplified in the high percentage of female Nobel prizes winners in 2020.

Workplace policies need also to be revised in terms of guaranteeing, first a proper remuneration of females working in the private sector and second a fair representation of women in all work positions, mainly senior ones.

Changing rules by implementing a tenure track policy could resolve part of this inequity. Indeed, this policy based on the use of a set of explicit and transparent criteria was applied at Wageningen University, The Netherlands (Bakker et al., 2016). As an outcome, chances on being promoted to higher levels have improved more for women than for men. However, the process is still slow because of the huge starting imbalance.

In addition to changing rules and policies, mindsets have to be changed. Mindset changes start from the educational system and include fostering leadership skills early within the education system. This was one of the recommendations 
advanced by Agili in her study on the unemployability among Tunisian graduates (Agili, 2020). We think that this recommendation can also be of great impact in preparing women for senior positions.

Governmental policies need to advocate for women by supporting them during their maternity leaves and create suitable framework conditions that take into consideration women specificities. This can include for example exceptional rules for women with 3 or more children aged below 14 years. Wendy M. Williams and Stephen J. Ceci in their study on the dearth of women in math-intensive fields have suggested several promising ways to increase women's representation in senior positions that focus on motherhood aspects such as couple culture change, adjustment of regulations that govern career development, and workspace fittings (Williams \& Ceci, 2012).

Government authorities are highly encouraged to grant women decision-making power by recruiting them into governance. All these initiatives need to be secured by laws and regulations to have sustainable and wide impact. To that regards, one of the alternatives that could increase the chance of a woman to gain a senior position is adding quotas. For example, mandate of no less than $35 \%-50 \%$ of women should be adopted in the parliament and in designated political positions.

The global situation, especially after the COVID-19 pandemic break out, has made the world more and more VUCA (Volatile, Uncertain, Complex and Ambiguous). Therefore nowadays, we cannot speak of stable or fixed recommendations to overcome the existing barriers since the world is in a continuous state of change.

\section{Conclusion}

If we are to take a revolutionary lens to humans, women are first and foremost mothers and play an important role in the stability of the family. Hence, nuanced attention should be paid when calling for an increase of women in the workforce. On the one hand, women need to have an opportunity to pursue their careers and dreams without compromising their biological role in reproduction and early childhood care. This is an important point that we highlight because there is a recent trend by some companies to encourage women to freeze their eggs to focus on their career and postpone their life as a mother to after the fifties if not after the retirement; rather than giving them time and support for this biological role within the biological clock. On the other hand, we need to ensure that there are enough women at the decision-making level to influence regulations and policies for a better representation and advocacy.

We must also keep in mind that many women are forced to work because of financial needs and don't have the luxury of choosing to work or stay at home.

\section{Acknowledgements}

To all my fellow scientists around the world:

This month, we celebrate the Nobel Prize winners. This is such an amazing 
and exciting event. I look forward to this week every year with my students. It's like the Oscars and more. This year was special because of all the women winners. I felt such a deep comradeship with them all. It's like I won as well. In reality, each one of us celebrates her own version of a Nobel Prize. It's not the grant you got or the paper you published or the award you received. Those are expected and guaranteed because you worked hard to achieve and knew eventually will get.

It's when your hypothesis holds out through testing and experiments, those moments that you experience with yourself, the gratification that you knew you were right is all captured there alone in your lab that you feel the thrill of the roller coaster in your body.

That is your Nobel Prize moment! The other achievements may happen or not. Others may know or they may not. It doesn't really matter because what matters is that you knew it all along whether it was acknowledged or not. So, seek answers, keep asking and stay curious. That is all that matters. Close your eyes, revel in your experience, failures and triumphs. You are the hero! The Nobel Prizes are a manifestation of who you are. We, scientists, are all a family, comrades and companions in the journey of discovery.

The quote was posted on social media by Rana Dajani in response to the Nobel Prize winners 2020.

\section{Conflicts of Interest}

The authors declare no conflicts of interest regarding the publication of this paper.

\section{References}

Agili, S. (2020). The Employability of the Tunisian Graduates. International Journal of Humanities and Cultural Studies, 7. https://ijhcs.com/index.php/ijhcs/article/view/3411

Bakker, M. M., \& Jacobs, M. H. (2016) Tenure Track Policy Increases Representation of Women in Senior Academic Positions, but Is Insufficient to Achieve Gender Balance. PLoS ONE, 11, e0163376. https://doi.org/10.1371/journal.pone.0163376

Ceci, S. J., Williams, W. M., \& Barnett, S. (2009). Women's Underrepresentation in Science: Sociocultural and Biological Considerations. Psychological Bulletin, 135, 218-261. https://doi.org/10.1037/a0014412

Clarke Whalen, E., Xu, G., Cygankiewicz, I., Bacharova, L., Zareba, W., Steinberg, J. S., Tereshchenko, L. G., \& Baranchuk, A. (2017). Gender Equity Imbalance in Electrocardiology: A Call to Action. Journal of Electrocardiology, 50, 540-542. https://doi.org/10.1016/j.jelectrocard.2017.05.002

Córdova, F. (2020). Leadership to Change a Culture of Sexual Harassment. Science, 367, 1430-1431. https://doi.org/10.1126/science.abb5791

Dajani, R. (2012). How Women Scientists Fare in the Arab World. Nature, 491, 9. https://doi.org/10.1038/491009a

Martin, A., Naunton, M., \& Peterson, G. M. (2021). Gender Balance in Pharmacy Leadership: Are We Making Progress? Research in Social and Administrative Pharmacy, 17, 
694-700. https://doi.org/10.1016/j.sapharm.2020.05.031

Ouyang, D., Sing, D., Shah, S., Hu, J., Duvernoy, C., Harrington, R. A., \& Rodriguez, F. (2018). Sex Disparities in Authorship Order of Cardiology Scientific Publications. Cir culation: Cardiovascular Quality Outcomes, 11, e005040. https://doi.org/10.1161/CIRCOUTCOMES.118.005040

Williams, W. M., \& Ceci, S. J. (2012). When Scientists Choose Motherhood: A Single Factor Goes a Long Way in Explaining the Dearth of Women in Math-Intensive Fields. How Can We Address it? American Scientist, 100, 138-145.

https://doi.org/10.1511/2012.95.138 